\title{
Usefulness of Magnetic Resonance Spectroscopy in the Assessment of Brain Chagas Disease: A Case Report
}

\author{
Angela Bernabeu ${ }^{\mathrm{a}, \mathrm{c}}$, Eduardo Alcaraz-Mateos ${ }^{\mathrm{b}}$, Susana Lopez-Celada ${ }^{\mathrm{a}}$
}

\begin{abstract}
Chagas disease, or American trypanosomiasis, is a parasitic infection caused by the flagellate protozoan Trypanosoma cruzi, an organism that is endemic to Latin America. While Chagas disease is primarily a vector-borne illness, new cases are emerging in non-endemic areas due to globalization of immigration and non-vectorial transmission routes. Once the infection has started, the main target organs are the heart, the alimentary tract, and the nervous system. However in immunosupressed patients, focal encephalitis due to T. cruzi infection may occur which tends to acquire a necrotizing feature with mass effect, perilesional edema, and pseudotumoral form (CTLAT, "cerebral tumor-like American trypanosomiasis"). In recent years, a few reports have described HIV-positive patients with central nervous system lesions due to Chagas disease. Here we report the clinical, neuroimaging, and histopathological findings of a patient with CTLAT and acquired immunodeficiency syndrome. To our knowledge, this is the first report in literature of the metabolite pattern assessed by magnetic resonance spectroscopy in these lesions.
\end{abstract}

Keywords: Central nervous system; Magnetic resonance spectroscopy; Brain; Abscess; AIDS

\section{Introduction}

Chagas disease, or American trypanosomiasis, is a parasitic infection caused by the flagellate protozoan Trypanosoma cruzi, an organism that is endemic to Latin America [1]. The

Manuscript accepted for publication October 15, 2013

${ }^{a}$ Magnetic Resonance Unit, INSCANNER SL, Hospital General Universitario de Alicante, Alicante, Spain

${ }^{b}$ Histopathology Unit, Hospital Universitario Morales Meseguer de Murcia, Murcia, Spain

${ }^{\mathrm{c}}$ Corresponding author: Angela Bernabeu, Unidad de Resonancia Magnetica, INSCANNER-ERESCANNER SALUD UTE, Hospital General Universitario de Alicante, C/Maestro Alonso 109, 03010

Alicante, Spain. Email: abernabeu@inscanner.es

doi: http://dx.doi.org/10.4021/jnr237w disease is transmitted to humans by a group of triatomine insects and less frequently by blood transfusion, congenital transmission, or contaminated organ transplants. While Chagas disease is primarily a vector-borne illness, new cases are emerging in non-endemic areas due to globalization of immigration and non-vectorial transmission routes [2]. Once the infection has started, the main target organs are the heart, the alimentary tract, and the nervous system. During the chronic symptomatic disease, abnormalities of the gastrointestinal tract (megaesophagus and megacolon) and/ or heart (cardiomegaly) are typically present [3]. However in immunosupressed patients, a focal encephalitis due to $\mathrm{T}$. cruzi infection may occur which tends to acquire a necrotizing feature with mass effect, perilesional edema, and pseudotumoral form (CTLAT, "Cerebral tumor-like American trypanosomiasis") [4]. In recent years, a few reports have described HIV-positive patients with central nervous system (CNS) lesions due to Chagas disease. Here we report the clinical, neuroimaging, and histopathological findings of a patient with CTLAT and acquired immunodeficiency syndrome. To our knowledge, this is the first report in literature showing the metabolite pattern of these lesions assessed by magnetic resonance spectroscopy (MRS).

\section{Case Report}

A 48-year-old male patient was hospitalized at the neurological unit after presenting a clinical history of increased headache, poor general condition, impaired vision, general fatigue, and dizziness. The patient was alert, oriented, and thin. He was from Argentina and did not present any personal or familiar antecedents of interest apart from smoking. The neurological and the general physical examination were normal except for a marked cachexia.

MRI examination revealed the presence of two brain lesions in the right hemisphere. One located in the frontal lobe measuring $12.4 \times 12 \mathrm{~mm}$, and the other located in the occipital lobe measuring $43 \times 26 \mathrm{~mm}$ (Fig. 1A). Both lesions appeared hypointense on T1-weighted images and hyperintense on T2-weighted and FLAIR images. No water diffusion restriction pattern was observed on the DWI im- 


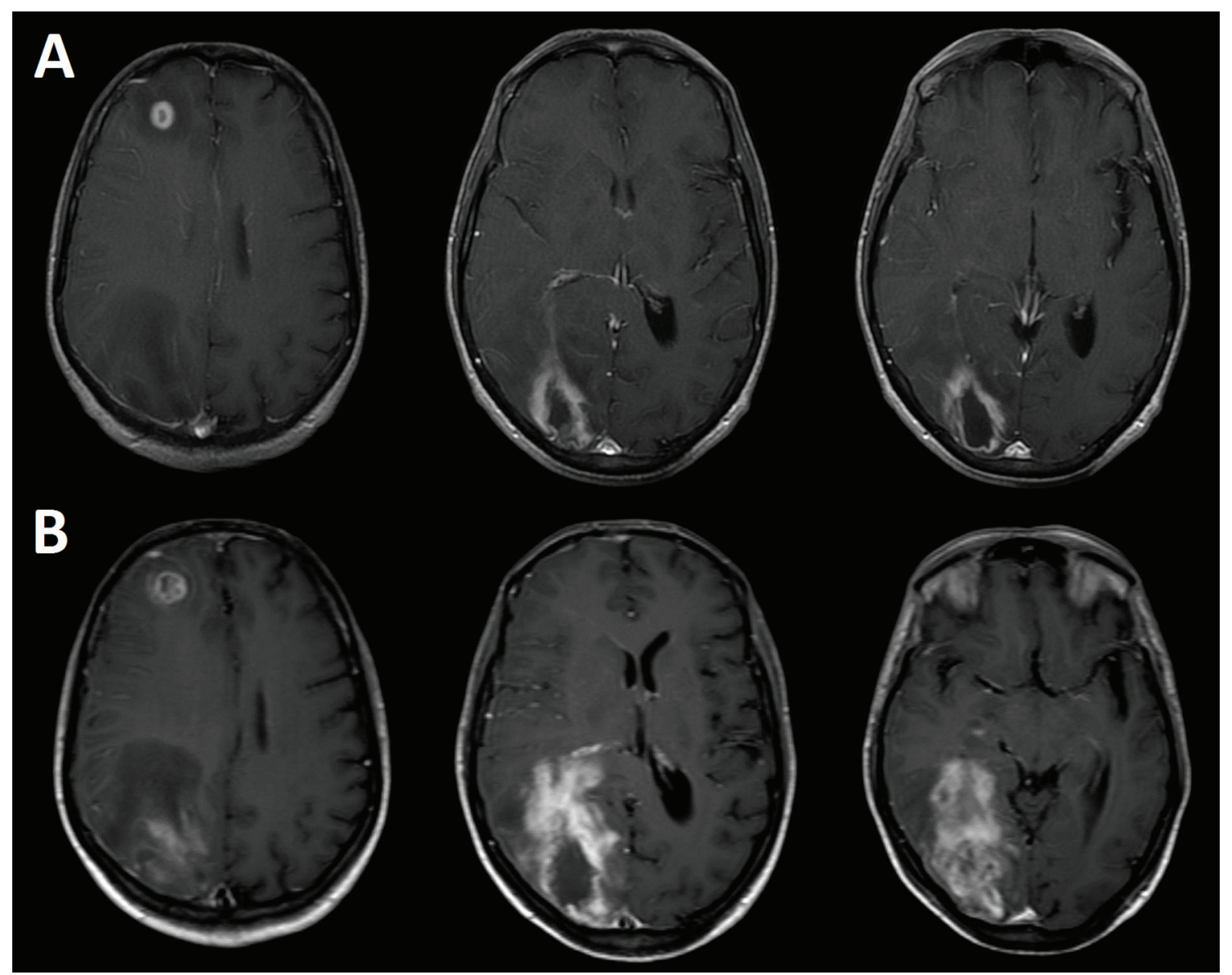

Figure 1. Contrast-enhanced (Gd-DTPA) axial brain T1-weighted images showing two cerebral lesions at the time of hospitalization (A), and 12 days after starting the anti-toxoplasmosis therapy (B). The images were acquired in a Philips Intera $1.5 \mathrm{~T}$ scanner with a standard quadrature head coil.

ages. After contrast injection (Gd-DTPA) ring enhancement was observed. Laboratory tests revealed VIH stage 3, being brain toxoplasmosis proposed in the differential diagnosis. The therapy with anti-toxoplasma drugs was started. Twelve days later, a second MRI study was carried out revealing the persistence of both lesions with a significant size increase (Fig. 1B). Specifically the frontal lesion was $18 \times 16 \mathrm{~mm}$ and the occipital lesion was $84 \times 35 \mathrm{~mm}$. MRS of both lesions revealed pathological metabolite changes with the presence of activity (increased choline), marked neuronal loss (decreased or absent NAA), and significant necrosis (presence of mobile lipids and/or lactate) (Fig. 2). The results showed the existence of marked neuronal loss even well outside the necrotic core (Fig. 2D). This metabolite profile, which was non-specific, could be related to several conditions of proliferation and/or inflammation.

Finally surgical brain biopsy of the occipital lesion revealed necrosis and chronic inflammation with the presence of macrophages with intracellular parasites (Fig. 3). The serology was positive for $\mathrm{T}$. cruzi (ELISA and IFI positives) and brain Chagas was finally diagnosed.

\section{Discussion}

Opportunistic infections with parasites are common in patients who have AIDS being Toxoplasma Gondii, the most common cause of focal brain lesions in these patients [5]. Although less common than T. gondii, T. cruzi also has the potential to cause fatal meningoencephalitis in patients with AIDS. States of immunodeficiency, such as human immunodeficiency virus infection, organ transplantation, or prolonged corticosteroid therapy, can result in the reactivation of the T. cruzi infection in chronically infected patients [4]. This is characterized by a high parasite proliferation and a clinical syndrome that resembles the acute phase. In fact, in some patients, chagasic encephalitis may be the first manifestation of AIDS [6]. Evidence of this involvement includes headaches, meningoencephalitis, seizures, lethargy, or mood changes.

In recent years, a few reports have described HIV-positive patients with CNS lesions due to Chagas disease [711]. In these reports, lesions appeared large-sized with lobar location and usually a thick ring enhancement surrounded 

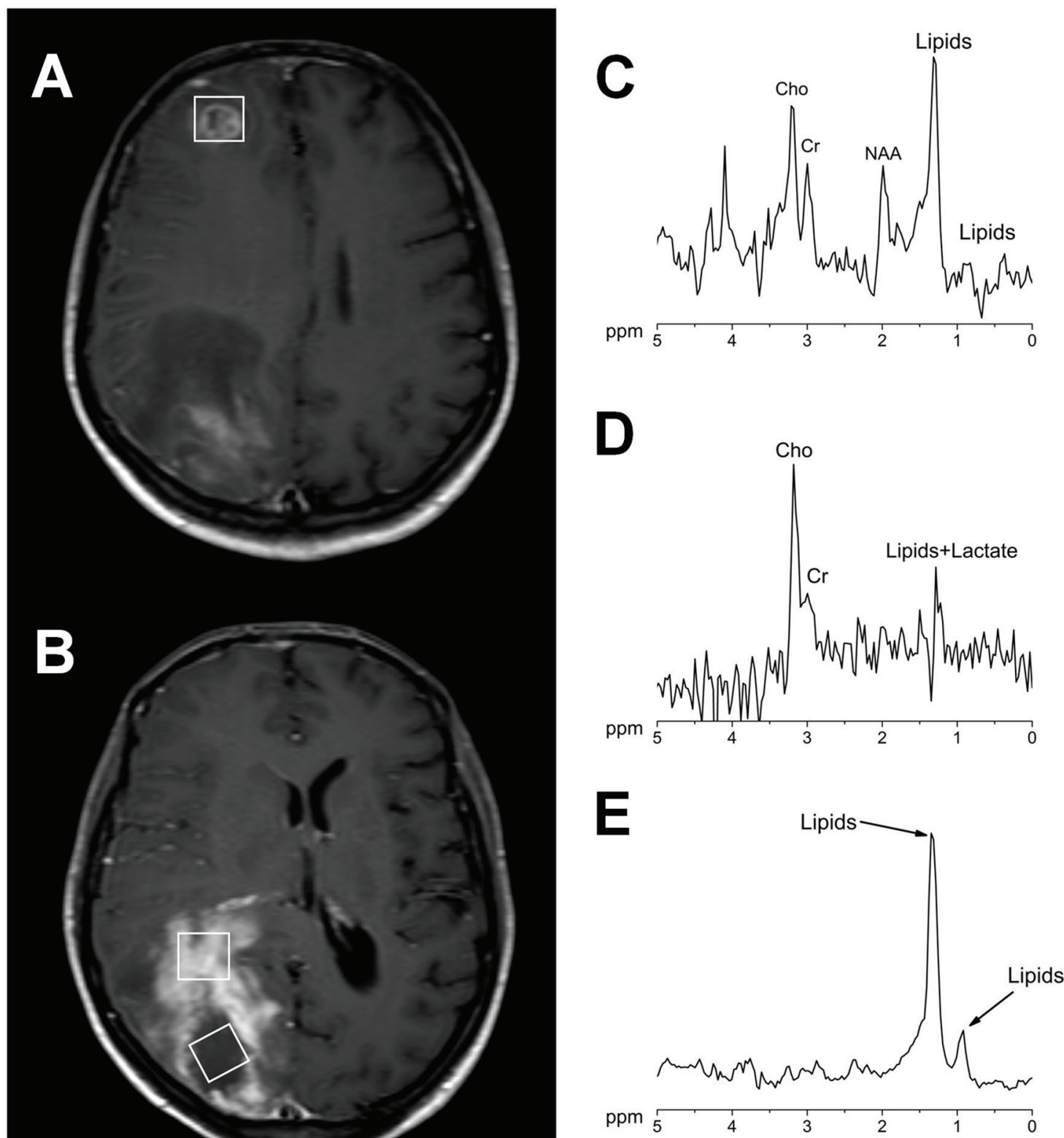

Figure 2. Contrast-enhanced axial brain T1-weighted images showing the voxel position in the measurement of both lesions (A, B). ${ }^{1} \mathrm{H}$ MR spectra acquired at $144 \mathrm{~ms}$ echo time from the right frontal lesion (C), the occipital lesion in the contrast agentenhancement area (D) and in the necrotic area (E). All the measurements were performed before the contrast agent injection in a Philips Intera 1.5 T with a standard quadrature head coil (PRESS/2000/144/128; Sequence/TR/TE/averages).

by severe edema. The clinical manifestations of these brain lesions are indistinguishable from other lesions also found in immunosuppressed patients, such as neurotoxoplasmosis, CNS lymphoma, and progressive multifocal leukoencephalopathy (PML). Some authors suggested that $\mathrm{T}$. gondii infections generally involve the cerebral cortex and the basal ganglia, whereas T. cruzi infections primarily affect the white matter [12]. However in our case, both lesions affected gray and white matter presenting an appearance indistinguishable from other brain lesions that usually appear in HIV patients
[13].

MRS complements conventional MRI by enabling better characterization of brain lesions with similar appearances on MR images and is increasingly being used in neurosurgical practices [14]. In our case, MRS showed a metabolite pattern which was slightly different for every lesion and depending on the voxel placement. Both lesions presented significant necrosis even for the frontal lesion which presented a smaller size. Findings related to inflammation and/or proliferation [15] and significant neuronal loss were also present. These 


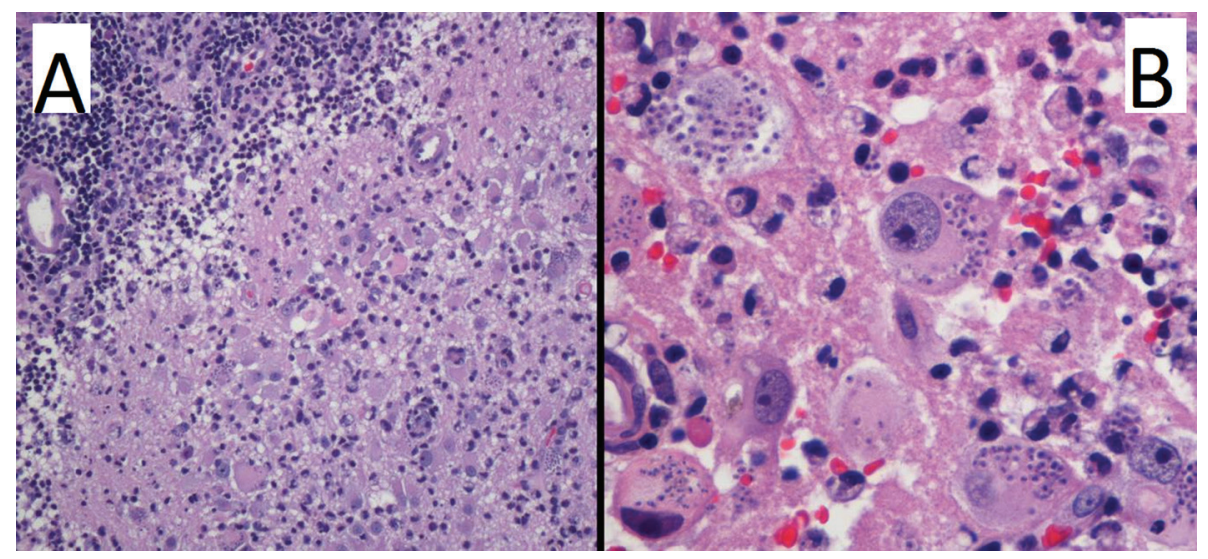

Figure 3. Hematoxylin and eosin stain at low (A) and high (B) power field. Images reveal brain parenchyma with necrosis and chronic inflammation (A), with the presence of lymphocytes and macrophages with intracytoplasmic parasites (B).

results are non-specific and consistent with different pathologies as brain lymphoma, PML, and other neoplasm such as glioblastoma, being unable to discard between these etiologies [16]. On the other hand, this metabolite pattern was very similar to the reported in brain toxoplasmosis $[16,17]$. This could be caused by the fact that both parasites induce inflammation and necrosis and therefore may share a similar metabolite profile.

Correct and rapid determination of the brain abscess agent is of value to more appropriately treat patients, as early anti-parasite treatment can improve the prognosis and survival of the patient. T. cruzi infection is a major cause of morbidity and mortality in endemic countries, but now, it is becoming a worldwide health problem as a result of urban and international migration. The involvement of the CNS is infrequent; however, it has a high mortality and morbidity rate especially when it is not well diagnosed and treatment is not quickly initiated $[2,18]$.

In this report, we describe a brain CTLAT showing that MRS displayed a metabolite pattern non-specific and similar to other brain pathologies. Although the MRS did not show a characteristic metabolite profile, this technique could be used to determine and better monitor the activity, neuronal loss, and necrosis degree of the lesions. In this context and, in addition to the neuroimaging findings, CTLAT should be strongly suspected in HIV-positive patients from endemic areas. Especially in patients that are not improving as rapidly as expected during anti-toxoplasmosis therapy.

\section{Acknowledgement}

Authors would like to thank Ms. Evelyn Teruel Sanchez for her outstanding technical support during the acquisition of the MR studies.

\section{References}

1. Abad-Franch F, Vega MC, Rolon MS, Santos WS, Rojas de Arias A. Community participation in Chagas disease vector surveillance: systematic review. PLoS Negl Trop Dis. 2011;5(6):e1207.

2. Perez-Molina JA, Rodriguez-Guardado A, Soriano A, Pinazo MJ, Carrilero B, Garcia-Rodriguez M, Salas J, et al. Guidelines on the treatment of chronic coinfection by Trypanosoma cruzi and HIV outside endemic areas. HIV Clin Trials. 2011;12(6):287-298.

3. Pittella JE. Central nervous system involvement in Chagas disease: a hundred-year-old history. Trans R Soc Trop Med Hyg. 2009;103(10):973-978.

4. Yoo TW, Mlikotic A, Cornford ME, Beck CK. Concurrent cerebral american trypanosomiasis and toxoplasmosis in a patient with AIDS. Clin Infect Dis. 2004;39(4):e30-34.

5. Collazos J. Opportunistic infections of the CNS in patients with AIDS: diagnosis and management. CNS Drugs. 2003;17(12):869-887.

6. Sica RE, Gargiullo G, Papayanis C. Tumour-like chagasic encephalitis in AIDS patients: an atypical presentation in one of them and outcome in a small series of cases. Arq Neuropsiquiatr. 2008;66(4):881-884.

7. Cohen JE, Tsai EC, Ginsberg HJ, Godes J. Pseudotumoral chagasic meningoencephalitis as the first manifestation of acquired immunodeficiency syndrome. Surg Neurol. 1998;49(3):324-327.

8. Lury KM, Castillo M. Chagas' disease involving the brain and spinal cord: MRI findings. AJR Am J Roentgenol. 2005;185(2):550-552.

9. Antunes AC, Cecchini FM, Bolli F, Oliveira PP, Reboucas RG, Monte TL, Fricke D. Cerebral trypanosomiasis and AIDS. Arq Neuropsiquiatr. 2002;60(3-B):730-733. 
10. Pagano MA, Segura MJ, Di Lorenzo GA, Garau ML, Molina HA, Cahn P, Perez H, et al. Cerebral tumor-like American trypanosomiasis in acquired immunodeficiency syndrome. Ann Neurol. 1999;45(3):403-406.

11. Del Castillo M, Mendoza G, Oviedo J, Perez Bianco RP, Anselmo AE, Silva M. AIDS and Chagas' disease with central nervous system tumor-like lesion. Am J Med. 1990;88(6):693-694.

12. Solari A, Saavedra H, Sepulveda C, Oddo D, Acuna G, Labarca J, Munoz S, et al. Successful treatment of Trypanosoma cruzi encephalitis in a patient with hemophilia and AIDS. Clin Infect Dis. 1993;16(2):255-259.

13. Gluckstein D, Ciferri F, Ruskin J. Chagas' disease: another cause of cerebral mass in the acquired immunodeficiency syndrome. Am J Med. 1992;92(4):429-432.

14. McLean MA, Cross JJ. Magnetic resonance spectroscopy: principles and applications in neurosurgery. $\mathrm{Br} \mathrm{J}$
Neurosurg. 2009;23(1):5-13.

15. Venkatesh SK, Gupta RK, Pal L, Husain N, Husain M. Spectroscopic increase in choline signal is a nonspecific marker for differentiation of infective/inflammatory from neoplastic lesions of the brain. J Magn Reson Imaging. 2001;14(1):8-15.

16. Kingsley PB, Shah TC, Woldenberg R. Identification of diffuse and focal brain lesions by clinical magnetic resonance spectroscopy. NMR Biomed. 2006;19(4):435-462.

17. Batra A, Tripathi RP, Gorthi SP. Magnetic resonance evaluation of cerebral toxoplasmosis in patients with the acquired immunodeficiency syndrome. Acta Radiol. 2004;45(2):212-221.

18. Ferreira MS, Nishioka Sde, Silvestre MT, Ferreira MS, Nishioka SA, Burgarelli MK, Almeida E, et al. Reactivation of Chaga's disease and acquired immunodeficiency syndrome. Am J Trop Med Hyg. 1994; 50: 261-268. 